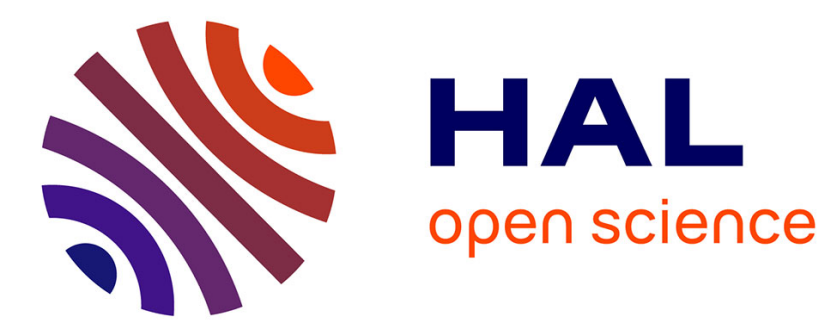

\title{
Structures avec constituants détachés et jugements d'évaluation
}

\author{
Agata Jackiewicz
}

\section{To cite this version:}

Agata Jackiewicz. Structures avec constituants détachés et jugements d'évaluation. Document numérique - Revue des sciences et technologies de l'information. Série Document numérique, 2010, Le texte, objet d'analyse et vecteur de connaissances, 13 (3), pp.13 - 40. 10.3166/DN.13.3.11-40 . halshs-01839138

\section{HAL Id: halshs-01839138 \\ https://shs.hal.science/halshs-01839138}

Submitted on 13 Jul 2018

HAL is a multi-disciplinary open access archive for the deposit and dissemination of scientific research documents, whether they are published or not. The documents may come from teaching and research institutions in France or abroad, or from public or private research centers.
L'archive ouverte pluridisciplinaire HAL, est destinée au dépôt et à la diffusion de documents scientifiques de niveau recherche, publiés ou non, émanant des établissements d'enseignement et de recherche français ou étrangers, des laboratoires publics ou privés.

\section{다)(1) $(5$}

Distributed under a Creative Commons Attribution - NonCommerciall 4.0 International 


\title{
Structures avec constituants détachés et jugements d'évaluation
}

\author{
Agata Jackiewicz \\ Equipe STIH, Université de Paris-Sorbonne \\ Maison de la Recherche \\ 28 , rue Serpente \\ F-75006 Paris \\ Agata.Jackiewicz@paris-sorbonne.fr
}

RÉSUMÉ. L'article présente une recherche portant sur des constituants détachés à caractère axiologique. L'approche choisie exploite et croise d'une manière originale les apports des travaux sur l'apposition, la sémantique de l'acte d'évaluation et les relations rhétoriques. Elle s'inscrit dans un projet mené à l'interface de la linguistique et du traitement automatique des langues (TAL), qui se donne pour but d'enrichir, d'adapter au français et de formaliser le modèle général Appraisal de l'évaluation dans la langue.

ABSTRACT. In this paper, we present a study about peripheral constituents concerning several axiological features. Our approach cross-examines and relies on results of research regarding apposition, semantics of evaluation and rhetorical relations. This study is a part of a larger project at the intersection of linguistics and NLP, the aim of which is to refine and formalize the Appraisal model of evaluation in language, and adapt it to the French language.

MOTS-CLÉS: jugements d'évaluation, constituants périphériques, constructions et lexiques axiologiques, relations sémantiques, Appraisal.

KEYWORDS: evaluative judgment, peripheral constituents, evaluative constructions and lexicon, semantic relations, Appraisal.

DOI:10.3166/DN.13.3.11-40 @ 2010 Lavoisier, Paris

RSTI - DN - 13/2010. Le texte : analyse et exploration, pages 11 à 40 


\section{Introduction}

L'article s'intéresse au phénomène textuel du détachement quand celui-ci sert de support à des jugements d'évaluation. Il cherche à mettre en évidence des régularités formelles et sémantiques qui caractérisent les structures avec constituants extraprédicatifs dédiées à l'expression des jugements ciblant des individus ou des institutions. De ce point de vue, notre travail constitue une contribution à l'étude des phénomènes évaluatifs dans la langue. L’apposition détachée, «poste fonctionnel destiné prioritairement à l'expression de l'analyse, de l'interprétation, du jugement » (Neveu, 1998), constitue un angle d'approche original et particulier, complémentaire des méthodes plus globales.

Les éléments périphériques à la prédication principale dénotent souvent des appréciations, sous forme réduite et clairement délimitée. On y trouve, sans exhaustivité, des expressions stéréotypées, figées ou semi-figées, des qualifications métaphoriques, des antonomases du nom propre, des adverbiaux d'attitude, des groupes adjectivaux (femme de tête, en bon élève, couronné de lauriers pour..., une main de fer, pur produit du sérail, à force d'obstination, avec courage, le Robin des bois du web, puriste certes, mais pas puritain...). Cette forme d'écriture que l'on rencontre dans des récits de $v^{1}{ }^{1}$ (biographies, portraits...) est également courante dans la presse (tant généraliste que spécialisée), dont sont issus nos corpus. Denses et faiblement modalisés, ces énoncés conviennent à l'expression des jugements établis ou relativement consensuels.

L'article, à dominante linguistique, présente une recherche en cours qui allie observations et analyses linguistiques avec expériences informatiques sur corpus. Ce travail prend place dans le cadre du projet ANR OntOpiTex ${ }^{2}$ qui vise la mise en place d'outils automatiques d'analyse sémantique fine des opinions et des jugements exprimés au sein de documents textuels numériques. En associant les regards de linguistes et d'informaticiens, d'universitaires et de professionnels de la veille, nous cherchons à proposer des modèles opératoires qui s'appuient sur une expertise de la langue (dont la construction fait l'objet de ce papier) et qui pourront déboucher sur des procédures d'extraction et de représentation des données d'opinion.

Le travail entrepris croise d'une manière originale plusieurs approches. Nous nous appuyons sur des recherches sur l'apposition, les constructions détachées et les compléments circonstanciels, décrites dans (Neveu, 1998, 2000 ; Guimier, 1996 ; Combettes, 1998). Au plan sémantique et énonciatif, nous empruntons l'appareil conceptuel de la théorie Appraisal (Martin et White, 2005). Du point de vue discursif, nous analysons les relations rhétoriques au sens de la Théorie de la structure rhétorique (Mann et Thompson, 1988) qui peuvent s'ancrer sur un jugement évaluatif. A terme, le but est de mettre clairement en évidence les critères d'intelligibilité et d'interprétabilité d'un jugement d'évaluation qui s'expriment à l'échelle du texte. Sur un versant plus

1. Voir (Neveu 1998).

2. Le projet OntOpiTex porte la référence ANR-08-CORD-009. 
formel, nous proposons des patrons linguistiques sous-jacents aux constructions et aux expressions axiologiques les plus saillantes, ainsi qu'un lexique constitutif de ces expressions. Sur cette base, on procède à des expérimentations informatiques afin de tester et d'amender les patrons, d'acquérir des lexiques et de collecter des observables. Ces ressources guideront l'analyse sémantique du fait évaluatif dans les textes des corpus applicatifs, et prépareront à l'implémentation des outils d'analyse d'opinions visés par le projet OntOpiTex.

Le croisement des domaines que nous revendiquons dans ce travail a pour conséquence l'impossibilité de présenter exhaustivement chacune des différentes problématiques impliquées : l'étude et le traitement des opinions, des affects et des appréciations, le système appositif, les opérations de détermination et de qualification, les relations rhétoriques. L'objectif de ce papier est plus modeste. En partant de l'analyse d'une famille d'expressions évaluatives en apposition, nous cherchons à identifier des dimensions saillantes des évaluations telles qu'elles fonctionnent dans des corpus choisis et en dégager des indices linguistiques. Les lexiques collectés sont soumis à des analyses fines pour dégager des catégories sémantiques dominantes, reflétant des systèmes de valeurs en usage. Ces résultats sont destinés à questionner le caractère opératoire et la couverture notionnelle de la théorie Appraisal, afin d'aboutir à un modèle linguistique adapté aux besoins du projet.

\section{Contexte, objet d'étude, observables et premières ressources}

\subsection{Contexte}

Ces dernières années ont été marquées par un intérêt croissant pour le traitement informatisé des opinions, des attitudes ou des sentiments exprimés dans des documents textuels, issus par exemple d'éditions en ligne des médias traditionnels ou de supports tels que les blogs ou les forums. Un nouveau champ de recherche propre s'est rapidement constitué autour d'équipes déjà nombreuses, poursuivant des objectifs divers et visant l'étude d'opinions portant sur des entités variées (produits, comportements...) sur la base de méthodes et de techniques non moins diversifiées. En témoignent entre autres l'apparition de nombreux ateliers et les récentes campagnes d'évaluation en fouille d'opinions FODOP'08, DEFT'07, DEFT'09, NTCIR6.

Une monographie assez complète sur le sujet a été réalisée par (Pang et Lee, 2008), présentant les différentes tâches que l'analyse d'opinion cherche à résoudre, les méthodes développées, ainsi que les applications existantes et potentielles de ces techniques. Les travaux informatiques relatifs aux phénomènes de l'évaluation traitent essentiellement de la fouille d'opinion et de l'analyse de sentiments. En simplifiant, on peut dire qu'ils s'inscrivent dans trois grandes catégories: (i) constitution de ressources lexicales ; (ii) classification de textes d'opinions ; (iii) analyse d'opinions dans les textes. 


\section{RSTI - DN - 13/2010. Le texte : analyse et exploration}

Dans la première famille, où l'objectif est de constituer des ressources lexicales de manière semi-automatique, les approches proposées se distinguent par le type de propriétés lexicales étudiées. Il s'agit par exemple de déterminer le caractère subjectif ou objectif des termes, statuer sur la polarité des termes subjectifs (positive, négative), ou encore préciser plus finement leur sens comme dans (Whitelaw et al., 2005) ou dans les travaux relatifs à SentiWordNet (Esuli et Sebastiani, 2006). Parmi les travaux du deuxième ensemble qui s'attachent à catégoriser des textes dans leur intégralité, citons par exemple les analyses de critiques de films (Turney, 2002; Pang, Lee, 2004) et une partie de DEFT07, de textes politiques ou de critiques de produits. Les techniques utilisées sont variées, majoritairement issues des domaines de la fouille de données et de l'apprentissage automatique. Dans la troisième famille, signalons les travaux qui extraient automatiquement des phrases porteuses d'opinion et permettent leur annotation manuelle avec une interface adaptée. Cette annotation se fait à l'aide des jeux d'étiquettes empruntées à des théories linguistiques qui proposent des modélisations fines du phénomène évaluatif. Par exemple, Bloom et al, (2007a ; 2007b) extraient les phrases porteuses d'opinions et les présentent dans un navigateur (Appraisal Navigator) qui offre à l'utilisateur la possibilité de choisir la catégorie Appraisal souhaitée, ainsi que différents paramètres comme la polarité, la force ou le type de la cible.

Sur le plan méthodologique, plusieurs points nous semblent importants à noter. On constate tout d'abord une grande diversité des approches de l'évaluation. Le domaine de la subjectivité étant encore faiblement balisé, on s'aperçoit que les chercheurs ne travaillent que rarement sur le même objet, même s'ils emploient la même terminologie. Le phénomène évaluatif est en fait vaste et multidimensionnel. Il se décline a minima en (i) attitude (elle-même susceptible de distinctions substantielles), (ii) polarité, (iii) intensité, (iv) cible et (v) source. Mais d'autres paramètres jouent également dans l'acte de l'évaluation (portée du jugement, engagement...). Par conséquent, différents niveaux de couverture et de précision peuvent être recherchés. Le deuxième point concerne une relative complexité des méthodes employées. On remarque que les démarches présentées se basent souvent conjointement sur des éléments théoriques (plus ou moins élaborés et abordés avec une fidélité variable), des choix pragmatiques (en fonction des contraintes applicatives), et s'appuient à des degrés variables sur l'existant (ressources, outils...). De ce fait, l'évaluation de la portée de ces travaux et la comparaison des résultats obtenus ne sont pas toujours aisées à réaliser.

Notons enfin que les travaux applicatifs les plus aboutis portent majoritairement sur les textes en anglais. Cela signifie que les ressources linguistiques, mais aussi les notions disponibles ne sont pas directement transposables pour le traitement des discours en français. Les architectures notionnelles élaborées renvoient à des systèmes de valeurs culturellement déterminés. Les lexiques couvrent des domaines restreints. Pour ce qui est de la théorie de Martin et White, par exemple, il s'agit, comme l'avouent les auteurs, du monde contemporain, occidental, postcolonial et australien. 


\subsection{La sémantique des jugements d'évaluation}

En linguistique française, la notion d'évaluation n'a pas encore reçu de description théorique unifiée. On note en revanche des recherches focalisées sur des notions choisies ou des ensembles de marques. En première approximation, semblent se détacher trois grands champs d'études ${ }^{3}$, non nécessairement disjoints, celui de l'opinion, celui de l'affect et celui de l'appréciation. L'opinion, qui dans son acception restreinte peut être vue comme «une forme inférieure de la connaissance» (Julliard 2008, p. 47), est de l'ordre de la conviction (Charaudeau, 1992). Identifiable grâce à des marques de points de vue (je crois que, je suis persuadé que, je trouve que...), l'opinion caractérise des énoncés où le locuteur exprime une position personnelle peu/ pas (encore) consensuelle. L'affect, assimilé à un « état psychologique », est de l'ordre du « ressenti » (Buvet et al., 2005; Anscombre, 2005). Une distinction significative à l'intérieur de ce champ trace une frontière entre des affects qui représentent une réponse émotionnelle à un stimulus extérieur (être surpris/étonné par qqch.) et des états affectifs non dirigés (être angoissé, peureux). On note que ce champ est différemment structuré selon les auteurs. Certains travaux aboutissent à des classifications raffinées, fondées sur plusieurs dizaines de classes sémantiques. Buvet et al., (2005) distinguent 63 classes pour les prédicats d'affect, dont $<$ amour $>$, $<$ colère $>$, $<$ joie $>$, <gaieté>, <haine $>$, <pitié>, <ressentiment>. Enfin, le vaste domaine de l'appréciation est, quant à lui, encore peu exploré au-delà des champs de l'éthique et de l'esthétique (dans leurs réalisations les plus évidentes: beau, bon, bien, juste). Charaudeau (1992) propose une organisation en quatre systèmes de valeurs : éthique, esthétique, hédonique et pragmatique, mais ne donne pas de critères linguistiques permettant de les circonscrire.

L'ouvrage Appraisal in English de (Martin et White, 2005), écrit dans le cadre de la linguistique systémique de Halliday, a attiré notre attention car il propose un modèle global, fondé sur une hiérarchie de notions. La théorie Appraisal distingue au premier niveau trois grands champs sémantiques : l'attitude, l'engagement et la gradation. L'attitude se décline en trois catégories: (i) l'affect, qui concerne les réactions émotives; (ii) le jugement, relatif à l'évaluation des personnes et de leurs comportements, et (iii) l'appréciation qui est l'évaluation des artefacts. Chacune de ces catégories articule à son tour une famille de valeurs sémantiques. Par exemple, le jugement inclut l'estime sociale (déclinée en normalité, capacité, ténacité) et la sanction sociale (où sont distinguées la véracité et la convenance). L'engagement regroupe les éléments qui indiquent de quelle façon le locuteur prend position sur le contenu de son discours ; cette catégorie a à voir avec le dialogisme (jugements monoglossiques/jugements hétéroglossiques). La gradation, enfin, rassemble les éléments qu'utilise le locuteur pour faire varier le degré d'impact de ses propos (la force relève de l'intensification, le focus concerne la typicalité). Les auteurs

3. La terminologie employée pour désigner ces champs est conventionnelle et encore peu stable. 
soulignent que cette architecture notionnelle a le statut d'hypothèse ${ }^{4}$ et que le modèle est ouvert à des aménagements. On doit à Monika Bednarek, notamment dans (Bednarek, 2009), des critiques très éclairantes sur certains aspects d'Appraisal, ainsi que des propositions d'aménagement pour ce modèle.

Pour rendre cette théorie opérationnelle dans notre perspective, il est nécessaire de vérifier, sur des corpus textuels applicatifs choisis pour le projet, (i) si les notions de l'Appraisal couvrent de manière satisfaisante les champs axiologiques qui y sont représentés ${ }^{5}$ et (ii) si la démarche d'assignation de ses différentes valeurs sémantiques peut être systématisée et au moins en partie rendue automatique.

\subsection{Constituants détachés à caractère axiologique}

A la frontière entre la phrase et le discours, les segments détachés constituent l'un des lieux privilégiés de l'expression des évaluations. Ces jugements peuvent porter sur des entités de tout type ontologique.

(1) Pur produit du sérail, l'inspecteur des finances est le parfait modèle de "l'honnête homme " cher à l'Hexagone : d'une courtoisie extrême, il est aussi à l'aise pour parler de finance internationale que de l'œuvre d'A. R. G6., dont il fut un proche.

Un constituant détaché, conformément à la définition qu'en donne (Combettes 1998, p. 136), est un «constituant qui ne se trouve pas intégré à la structure syntaxique de la proposition, qui n'entre pas dans des relations de dépendance ou de rection avec d'autres éléments ». Selon sa nature (adjectivale, nominale, adverbiale...), un tel constituant satisfait à un certain nombre de critères, dont les principaux sont: délimitation par des signes de ponctuation, possibilité d'effacement, liberté de position, statut de prédication seconde, existence d'un référent sous-jacent... Non essentiel, il est en réalité plus ou moins nécessaire (effaçable) et plus ou moins libre (déplaçable). Occupant une place parfois instable entre phrase et texte, les constituants périphériques à la prédication principale peuvent franchir les limites de la phrase graphique (2), tout en restant sémantiquement liés à celle-ci.

4. « Notre cartographie des émotions (relative à l'affect, au jugement et à l'appréciation) doit être considérée comme une hypothèse concernant l'organisation des significations sousjacentes. Offerte comme un défi à ceux qui s'intéressent au développement des raisonnements appropriés, comme un élément de référence pour ceux qui proposeraient des classifications alternatives, et enfin comme un outil pour ceux qui mènent des analyses de l'évaluation dans le discours. » (Martin et White, $2005: 46$ ). C'est nous qui traduisons.

5. On peut s'attendre par exemple à ce que le domaine de l'économie que nous sommes amenés à traiter entraîne des aménagements spécifiques, notamment pour représenter des notions telles que efficacité et utilité.

6. Dans les exemples, les noms des personnes ont été substitués par leurs initiales. 
(2) Car lors de ce grand raout, ce n'est pas lui, mais A. M., qui triomphera. En silence. Discrètement, comme à son habitude. Mais avec une belle assurance. Celle des vainqueurs sûrs de leur fait, de leur force tranquille.

Les constituants périphériques peuvent dénoter des propriétés objectives ou subjectives, à l'axiologie stable ou instable. Leur interprétation appartient au lecteur, amené à décider en fonction de son point de vue et son propre système de valeurs ${ }^{7}$.

Les constituants détachés potentiellement axiologiques peuvent être regroupés, en première approximation, en trois catégories : celle des constructions détachées, celle des compléments circonstanciels et celle des groupes nominaux déterminés ou non (3-5). La catégorie de construction détachée, au sens de (Combettes, 1998), intègre les groupes adjectivaux (imprévisible et fantasque, X...), les constructions absolues (l'œil vigilant, X...) et les participes (réputé pour son caractère bourru, $\mathrm{X} . .$.$) . La seconde famille de constituants périphériques aptes à dénoter des$ évaluations est formée par des adverbiaux orientés sujets (d'attitude ou de manière), (Guimier 1996 : 69-103) et par des circonstants prépositionnels (Habilement, X... ; En mauvaise posture, $X$ ). Enfin, le troisième ensemble de constituants périphériques comprend des groupes nominaux avec ou sans déterminants tels que homme de convictions, $X \ldots$ ou $X$, le maestro de la désinflation.

(3) Pragmatique et prudent, B.O. s'est bien gardé jusqu'ici de chiffrer son plan de relance dans les infrastructures.

(4) Le conseil des impôts a décidé, très sagement, de supprimer celles dont les effets sont impossibles à évaluer.

(5) Patron respecté, il n'a pas su se faire aimer par ses troupes.

Les constituants extra-prédicatifs jouent un rôle dans la hiérarchisation des informations ${ }^{8}$. De l'ordre de la digression ou du présupposé, les contenus qu'ils dénotent sont généralement relégués au second plan, car ils sont vus comme acquis ou relativement consensuels. Cela a pour effet de minimiser la responsabilité énonciative de l'auteur. De plus, si leur rapport avec le reste de la phrase n'est pas marqué explicitement (ce qui est généralement le cas), son interprétation reste ouverte. Par exemple dans (6), le rapport causal entre l'arrogance de la compagnie en question et son échec commercial n'est pas explicité. Mais cette juxtaposition suffit pour suggérer une telle lecture.

7. «Passer de l'énumération des propriétés objectives d'un objet à son évaluation axiologique, c'est effectuer, toujours, en prenant appui sur ses compétences culturelle et idéologique, un certain 'saut interprétatif' (plus ou moins audacieux, plus ou moins contestable) » (Kerbrat-Orecchioni, 2002, p. 107).

8. Au chapitre de la structuration des contenus, notons également que certains constituants adverbiaux en position frontale pourraient ouvrir des cadres de discours. Ce point tout à fait important, que nous n'avons pas encore exploré, mérite une étude en soi. 
(6) Volontiers arrogant, mal remis du scandale de corruption de 2004, trop sûr de son fait, l'avionneur de Seattle s'est fait battre à plates coutures par un outsider d'autant plus motivé qu'il joue son entrée sur le premier marché $d u$ monde.

L'écriture avec constituants détachés est couramment pratiquée dans la presse, car elle est condensée, fluide et dynamique, propice à des effets de style (7). On note que les articles à vocation biographique (portraits, rubriques de type " en vue », nécrologies...) abondent d'énoncés à constituants détachés multiples. On le sait, les segments détachés (adjectivaux, adverbiaux, sous forme de constructions absolues...), dès lors qu'ils remplissent des fonctions similaires, se trouvent combinés sans difficultés. Ce sont de véritables condensés de qualifications ou d'appréciations permettant de brosser en peu de mots des portraits vivants, détaillés et nuancés. «Le souffle est court, le rythme alerte, le ton vif, les effets de densité et de resserrement sont très accentués » (Neveu, 1998).

(7) Un esprit pointu dans un corps rond, une grosse tête qui ne s'embarrasse pas d'académisme même s'il siège à l'Académie des sciences, un bourreau de travail plutôt sérieux doublé d'un conteur d'histoires désopilant, un " malgré lui " de la politique qui dit chaque fois qu'on ne l'y reprendra plus, CA est un personnage. Couvert de gloire scientifique pour ses travaux de géochimie (il a reçu la médaille d'or du CNRS, et surtout le prix Crafoord, considéré comme un équivalent du Nobel), incollable sur la datation de chaque pli du manteau terrestre, ce chercheur qui trouve est aussi un fidèle au culte de l'amitié. (...)

\subsection{Amorçage de l'analyse}

\subsubsection{Patrons, lexiques, attestations}

L'étude linguistique de ces constituants, préalable à toute expérimentation informatique, a été menée sur les textes du journal d'économie les Echos en accès libre sur www.lesechos.fr. L'exploration de ces textes a été manuelle; elle a été menée en trois temps. La première étape a consisté à prélever systématiquement tous les énoncés répondant aux critères retenus dans les textes des rubriques «En vue » et «Portraits» (110 textes). Lors de la deuxième étape, nous avons recherché sur l'ensemble du site des occurrences des marques (traduites en français) associées à la catégorie «Jugement» dans la théorie Appraisal (Martin et White, 2005, p. 53) en prélevant les énoncés où les marques en question figurent dans un constituant détaché. Sur la base de ces attestations nous avons formulé un premier ensemble de patrons linguistiques (une dizaine environ). Dans un troisième temps, nous avons recherché, toujours sur l'ensemble du site, certaines configurations précises de marques dans le but de systématiser ces patrons. A chaque étape, les marques lexicales constitutives des segments évaluatifs étudiés ont été collectées et organisées dans des classes selon leur catégorie grammaticale et leur fonction dans le segment. 
Les régularités observées mettent en évidence l'existence de différentes formes de surface, des constructions pouvant être précisées par des lexiques spécifiques. Nous avons ainsi dégagé 20 patrons linguistiques sous-jacents aux expressions axiologiques les plus saillantes, ainsi qu'un lexique (550 termes) constitutif de ces expressions (Jackiewicz, 2009). Un corpus d'exemples associés aux patrons linguistiques a également été créé. Aucune forme d'exhaustivité n'est revendiquée à ce stade de l'étude. Chaque patron nécessite à son tour une étude linguistique approfondie. A ce jour, nous avons analysé sur corpus quatre de ces patrons (P1, $\mathrm{P} 11, \mathrm{P} 16$ et P17). On trouvera dans les sections qui suivent quelques résultats qualitatifs et quantitatifs issus de ces analyses.

\begin{tabular}{|c|}
\hline $\mathrm{P} 1:\{$ homme, femme $\}$ de $\mathrm{N}$-femme de caractère - génitif de qualité \\
\hline $\mathrm{P} 2$ : (art)\{homme, femme\} à $\mathrm{N}$ - un homme à la forte personnalité - épithète \\
\hline P3 : art N Adj-l'obstination bretonne - construction absolue (pour les titres) \\
\hline P4 : art \{oeil, nez, tête, visage... $\}$ Adj - l'œil vif - constr. abs. avec parties du corps \\
\hline P5 : $\{$ virtuose, maestro, ... $\}$ de GN - maestro de la désinflation - expr. métaphorique \\
\hline P6 : $\mathrm{N}$ adj / adj $\mathrm{N}$ - économiste brillant - GN sans dét. - < savoir-faire > \\
\hline P7 : \{fine mouche, petit soldat, canard boiteux...\} - expressions figées métaphoriques \\
\hline $\mathrm{P} 8$ : art NP de GN - un Rimbaud de l'ère numérique - antonomase du nom propre \\
\hline P9 : \{parangon, modèle... \} de GN - parangon du cynisme \\
\hline P10 : (Adj) \{adepte, chantre, amateur,...\} de (Adj) $\mathrm{N}$ - amateur de bonne chère \\
\hline P11 : GAdj-imprévisible et fantasque - groupe adjectival \\
\hline P12 : GAdj à/pour Vinf - habile à construire...- groupe adj. à expansion infinitive \\
\hline P13 : de artInd Adj N / N Adj - d'une extrême fermeté - génitif de qualité \\
\hline P14 : de art-ind \{voix, geste, regard, œil... $\}$ Adj - d'un regard ironique \\
\hline P15 : \{fort, fier, riche...\} de art_poss (Adj) $\mathrm{N}$-fort de ses succès - <expérience> \\
\hline P16 : \{réputé, salué... \} pour art_poss (Adj) N - réputé pour son sang froid - participe \\
\hline P17 : en N Adj / Adj- en industriel responsable - structure prépositionnelle en en \\
\hline P18 : \{avec, sans, non sans $\}$ N/ adverbe en 'ment' - avec pragmatisme - adverbial \\
\hline P19 : \{grâce à, à force de...\} (art_poss) N - à force d'obstination - locution causale \\
\hline
\end{tabular}

Tableau 1. Patrons linguistiques avec leurs catégories et illustrations

\subsubsection{Patrons : caractéristiques formelles et sémantiques}

Les patrons identifiés, tels qu'ils sont représentés dans le tableau 1, renvoient à des formes de surface récurrentes, mais non nécessairement stables. En effet, la plupart de ces patrons peuvent admettre des variantes (dues à la présence/l'absence d'adjectifs, d'adverbes, d'expansions en que/qui, de coordinations). Ces patrons ne 
correspondent donc pas à des constructions maximales. Par exemple, le groupe adjectival (P11) se décline en une multiplicité de formes, plus ou moins complexes (populaire; imprévisible et fantasque; ni honnête, ni digne de confiance; charmeur sans être hâbleur ; arrogant et sans scrupule; plus instinctif qu'érudit ; vrai rebelle et faux intrépide...).

Les patrons correspondent à des expressions à sens souvent compositionnel, même si des formes figées en font également partie, saturés par des lexiques à l'étendue variable. En effet, certaines constructions semblent servir, notamment dans des médias, de tours "prêts à l'emploi ", avec des lexiques routiniers (voir section 3.2).

Le tableau 1 présente les patrons linguistiques dégagés sans y introduire de catégorisation. Or, il est possible de les regrouper selon différents ordres de critères.

Tout d'abord, selon la nature de leurs composants, on distingue (i) des patrons génériques constitués de catégories grammaticales et de prépositions ( $\mathrm{P} 3, \mathrm{P} 6, \mathrm{P} 8$, P11, P12, P13, P17), (ii) des expressions figées construites sur un modèle particulier (P7, P20), (iii) des patrons faisant appel à des lexiques plus ou moins spécifiques, plus ou moins étendus (tous les autres patrons). De ce fait, les règles correspondantes, utilisées pour la recherche des patrons dans des corpus, ne sont pas toutes comparables. Les règles qui exploitent des listes d'entrées lexicales finies pourront entraîner du silence, d'autres, plus génériques, sont susceptibles d'amener $\mathrm{du}$ bruit. Cette question de précision et d'ambiguité doit être considérée indépendamment pour chaque patron. En fonction de l'objectif de la recherche, de la taille du corpus et des ressources lexicales disponibles un réglage précis pourra et devra être effectué (voir section 2.4.3). Par exemple, le recours à des marques contextuelles supplémentaires, et notamment à celles qui renvoient à la cible du jugement (sous forme de nom propre ou de pronom personnel), peut être envisagé.

En deuxième lieu, si l'on reprend la classification plus globale des constituants détachés possiblement axiologiques (section 2.3), nous avons des patrons qui entrent dans (i) la famille des constructions détachées (adjectivales, participiales, de type construction absolue) (P3-P4 ; P11-P16), (ii) dans l'ensemble des formes adverbiales et apparentées (P17-P20), (iii) dans la classe des constituants nominaux (P1-P2 ; P5P10). Les différences de fonctionnement sémantique entre ces trois catégories de patrons sont notables. Par exemple, la famille des adverbes et des formes prépositionnelles se distingue des deux autres par son rôle essentiellement circonstanciel. Elle présente la situation dénotée par la prédication principale comme une manifestation d'une qualité attribuée à l'actant support. La portée de l'évaluation est alors restreinte à la situation décrite (action, comportement...).

Enfin, le dernier critère renvoie au type (ontologique et/ou sémantique) de la cible de l'évaluation. Certains patrons capturent des qualités spécifiquement humaines, d'autres, formés de catégories grammaticales et de prépositions, peuvent s'appliquer à des cibles de différentes natures. Dans le premier ensemble notons : 
- des patrons qui contiennent un terme catégorisant spécifique (P1, P2, P5, P10...) femme de tête, apôtre du capitalisme familial...

- des patrons qui exploitent le langage du corps humain (P4, P14) une main de fer, un rire de velours, l'œil vif...

- des antonomases du nom propre (P8) : ce procédé fournit un personnage connu avec qui l'identification fait apparaître une qualité, une manière d'agir ou un style de comportement remarquable, éventuellement transposé ou corrigé : un Rimbaud de l'ère numérique, une sorte de Rachida Dati des affaires et au masculin...

\subsubsection{Ajuster les patrons : ambiguïté et couverture}

La question de la fiabilité des patrons, liée à celle des conditions précises dans lesquelles ces structures ramènent des résultats escomptés, est à la fois centrale et complexe. Pour vérifier l'impact de certains paramètres (ponctuation, position, marques de la cible) sur la couverture et la précision des patrons, nous avons réalisé plusieurs recherches sur l'intégralité de la base catégorisée Frantext ${ }^{9}$. Le tableau 2 présente quelques requêtes Frantext, réalisées pour les patrons P6, P13 et P1. Précisons que l'interprétation des résultats implique deux ordres de décision: (i) s'agit-il d'un constituant détaché ? Car certaines configurations ponctuationnelles sont ambiguës, (ii) s'agit-il d'un segment évaluatif? Il s'agit donc d'une tâche très lourde.

\begin{tabular}{|c|c|c|c|}
\hline Règle & Requête Frantext & $\begin{array}{l}\text { Nombre total } \\
\text { d'attestations }\end{array}$ & $\begin{array}{l}\text { Nombre/ pourcentage } \\
\text { d'attestations } \\
\text { correctes }\end{array}$ \\
\hline R6_1 & $(.||-,\mid$ ?!!!:|;) \&e(g=S) \&e(g=A) (.I,I-|?|!!:|;) & ?? & \\
\hline R6_2 & (.|?|!) \&e(g=S) \&e(g=A) (, ) \&e(g=Np) & 79 & $24(30 \%)$ \\
\hline R6_3 & $(.|?| !) \& e(g=S) \& e(g=A)(,$,$) (illelle|ils|elles)$ & 196 & $60(30 \%)$ \\
\hline R6_4 & (.|?|!) \&e(g=A) \&e(g=S) (, ) \&e(g=Np) & 90 & $17(19 \%)$ \\
\hline R6_5 & (.|?|!) \&e(g=A) \&e(g=S) (, ) (illelle|ils|elles) & 247 & $108(44 \%)$ \\
\hline R13_1 & $(.|?| ! \mid),\left(d^{\prime}\right.$ un|d'une) \&e(g=S) \&e(g=A) (, ) & 4451 & \\
\hline R13_2 & $(.|?| ! \mid),\left(d^{\prime}\right.$ un|d'une) \&e(g=S) \&e(g=A) (, , ) \&e(g=Np) & 79 & $69(87 \%)$ \\
\hline R13_3 & $(.|?| ! \mid),\left(d^{\prime} u n \mid d^{\prime} u n e\right) \& e(g=S) \& e(g=A)(,$,$) (illelle|ils|elles)$ & 435 & $388(89 \%)$ \\
\hline R1_1 & 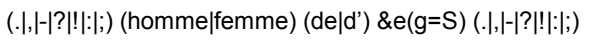 & 251 & $251(100 \%)$ \\
\hline R1_2 & $(.||-,\mid$ ?!!|:|;) (homme|femme) (de|d') \&e(g=S) & 500 & $500(100 \%)$ \\
\hline R1_3 & (homme|femme) (de|d') \&e(g=S) (.|,|-|?|!|:|;) & 4127 & \\
\hline R1_4 & (homme|femme) (de|d') \&e(g=S) & 8751 & \\
\hline
\end{tabular}

Tableau 2. Requêtes Frantext pour les patrons P1, P6 et P13 
Considérons tout d'abord le patron générique P6 « $(\mathrm{N} \operatorname{Adj}) \mid(\operatorname{Adj} N) »$. La fréquence de ce patron en position détachée dépasse 25000 occurrences (pour une recherche accomplie à $30 \%$ ). Une configuration spécifique, à l'initiale absolue, avec à droite du constituant une occurrence d'un pronom personnel ou d'un nom propre, en qualité du marqueur du support, renvoie au total 612 attestations, dont un tiers correspondent fidèlement à la structure que nous recherchons. En effet, dans $34 \%$ des cas, nous y trouvons un terme qui indique la catégorie d'appartenance de la cible associée à un adjectif dénotant une qualité (compositeur fécond, politique médiocre, homme illustre, pêcheur astucieux, homme universel, peintre sublime, chercheur intrépide... ou intrépide marin, pauvre fille, savant géologue, honnête homme...).

La démarche analogue réalisée pour le patron P13 (présent en apposition 4451 fois) permet de montrer que l'association avec des marqueurs de la cible ramène 514 résultats, dont $88 \%$ correspondant à la configuration recherchée ( $d$ 'un regard sévère, d'un geste furibond, d'un air grave, d'une voix patiente, d'un bond gracieux, d'une main méprisante...). On constate que ce tour semble se spécialiser pour dénoter une attitude corporelle (bond, geste, pas...) caractérisant un actant en action. On note que 141 attestations $(27 \%)$ contiennent la séquence «d'un geste...».

Le patron P1, enfin, fortement spécifié par les deux termes homme et femme, suffit pour identifier des séquences pertinentes. Il est présent en apposition 251 fois et 8751 fois quand les contraintes de ponctuation sont levées entièrement. Dans le deuxième cas, il est nécessaire de vérifier si et comment la position argumentale influence la sémantique de cette construction.

\section{Premières analyses sur corpus}

Cette première analyse manuelle du phénomène évaluatif sur le corpus des Échos a permis de proposer un ensemble de patrons récurrents ainsi qu'un lexique associé. A partir de ces ressources, plusieurs tests informatiques sur corpus analogues ( Le Monde) ont été réalisés ou sont en cours, en collaboration avec Thierry Charnois et Stéphane Ferrari (Jackiewicz et al. 2009 a ; 2009 b ; Ferrari et al. 2009). Ces tests et explorations ont porté sur 11 patrons. Les patrons linguistiques ont été implémentés à l'aide d'expressions régulières écrites en Perl. Dans ce qui suit, nous présentons quelques analyses ciblées, appuyées par des statistiques.

\subsection{Collectes ciblées des observables}

Le patron linguistique qui a permis de collecter le plus grand nombre d'observables est le groupe adjectival (P11) : 1662 attestations dans 535 articles de 
catégorie portrait ou biographie du Monde (sur un total de $884 \operatorname{articles}^{10}$ ). Grâce à l'examen de ces données, nous avons pu identifier plusieurs phénomènes significatifs qui deviendront autant de pistes de recherche pour la suite du projet : (i) présence significative d'énoncés à constituants détachés multiples (énumérations) et de groupes adjectivaux complexes (8), (ii) jeux d'opposition ou de renforcement par la présence de connecteurs, (iii) phénomènes de contagion contextuelle influençant la polarité (Tenace, solide, décidé, mordant.), (iv) présence de groupes adjectivaux autonomes, (v) influence de la position détachée sur l'interprétation axiologique de certains adjectifs (ouvert, européen).

(8) Chaleureux, provocateur, soupe au lait, terriblement affectif, M.K. tranchait dans un monde patronal souvent conventionnel.

Une analyse fine des 100 premières attestations par Zerguini, (2009) a permis de confirmer ces tendances. $5 \%$ des groupes adjectivaux sont graphiquement autonomes. $60 \%$ des énoncés comportent un groupe adjectival composé. Dans ces 100 attestations, on dénombre 153 éléments axiologiques. Ces résultats montrent que les jugements évaluatifs portés sur des personnes revêtent souvent un caractère analytique (adapté à l'expression d'appréciations focalisées, nuancées...). Cette propriété est à prendre en considération pour la mise en place de traitements informatiques futurs, notamment pour la délimitation des segments minimaux dénotant des jugements et pour le calcul de la polarité de ces jugements.

Sur le plan sémantique, la catégorisation de ces lexiques constitue une tâche difficile, car la frontière est souvent floue ou ténue entre l'acte de qualification et l'acte de jugement axiologique. Le modèle Appraisal a permis de typer de manière sûre seulement 83 items. L'examen approfondi des termes inclassables avec Appraisal demande à être poursuivi. Toutefois, nous pouvons d'ores et déjà donner quelques éléments explicatifs. Le jugement de type « sanction sociale » correspond à l'évaluation des comportements par rapport à des normes sociales codifiées par des lois, des codes, des règlements qui dictent comment se comporter. La catégorie «l'estime sociale » correspond quant à elle à l'évaluation des comportements par rapport à des expectatives sociales ou des systèmes de valeurs moins bien définis, présents implicitement dans la culture. Cette distinction intuitivement satisfaisante, s'avère en pratique difficile à appliquer, particulièrement quand le domaine (culturel, professionnel...) concerné par le texte est peu (ou pas) connu de l'interprétant. Ce dernier cherche alors à s'appuyer sur les notions « filles » (normalité, capacité...) pour classer les qualités. Or, les rubriques proposées ne couvrent pas tous les champs possibles. Par exemple, des attitudes sociales qui échappent à la «sanction sociale» (consensuel, conciliant...) demeurent inclassables, dès lors qu'elles ne tombent pas sous les concepts spécifiques de normalité, de capacité ou de ténacité. Le deuxième point qui émerge est la question de la pertinence de certains choix opérés par les auteurs de la théorie Appraisal. Pourquoi réserver la notion de normalité au jugement

10. La taille de ce corpus représente 4 Mo (environ 700000 mots). 
des cibles humaines? Celles de l'impact et de l'estimation à l'appréciation des artefacts? Ces questions ouvrent autant de pistes de recherches futures.

\subsection{Acquisition de lexiques}

Certains patrons peuvent être utilisés pour acquérir des lexiques axiologiques sur des corpus choisis. Nous avons réalisé une étude linguistique approfondie (vérification du taux d'ambiguité, recherche de variantes formelles, collecte des lexiques, analyse en champs sémantiques...), sur 16 ans $d u M_{\text {Monde }}{ }^{11}$, pour deux d'entre eux : P1 et P16.

La démarche générale d'acquisition de lexiques pour ces deux patrons est incrémentale. Elle consiste à fixer la partie gauche du patron (la tête), formée par une amorce de lexèmes, pour collecter le lexique de la partie droite (l'expansion), puis selon le degré de figement du patron, s'appuyer sur le lexique acquis de la partie droite pour enrichir les constituants de gauche. Montrons de manière synthétique quelques résultats obtenus pour le patron P16.

Pour l'expérimentation informatique, nous avons décliné cette construction en trois variantes sémantiquement proches :

- P16_1 : \{réputé, connu, salué, reconnu, admiré, remarqué, apprécié, célébré, renommé, encensé, critiqué, sanctionné, acclamé, distingué\} pour art_poss GN

- P16_2 : \{réputé, connu ...\} comme GN

- P16_3 : \{réputé, connu ...\} pour être GAdj/GN.

Les listes des participes passés ont été construites manuellement lors de la première phase de l'étude (section 2.4.1). La recherche de ces trois constructions sur le corpus du Monde a ramené au total 590 attestations, avec la distribution suivante P16_1 : 546, P16_2 : 26, P16_3 : 18. Parmi les 590 énoncés, 171 (29\%) ne portent pas sur des individus, mais concernent des lieux, des artefacts ou des produits naturels (réputée pour son patrimoine architectural, renommée pour son architecture baroque, réputé pour ses combats de taureaux, réputé pour ses pages sportives, connu pour son classicisme, réputé pour sa beauté...). Le cas des artefacts mérite toutefois attention, car ils participent à la réputation des entreprises qui les fabriquent ou les diffusent. Parmi les attestations qui renvoient à des individus, $82 \%$ concernent le genre masculin.

L'analyse des composants lexicaux de cette construction révèle une nette tendance à privilégier certaines expressions, faisant émerger des formules de routine.

Les participes connu et réputé figurent dans $84 \%$ des attestations. Une expérience inverse visant l'acquisition automatique de participes pouvant figurer en tête de cette construction a été menée sur le même corpus, à partir d'une sélection

11. 16 ans du Monde (1987-2002) : 5,5 Go (environ 870 millions de mots). 
d'extensions attestées dans la première étape d'extraction. Elle a ramené seulement 3 nouveaux participes condamné, redouté, décoré, attestés chacun une seule fois (décoré pour ses actions au Vietnam, redoutée pour ses jugements lapidaires, condamné pour ses positions coperniciennes).

L'analyse des expressions récurrentes figurant dans l'expansion montre que cette construction est employée pour mettre en relief une caractéristique saillante et valorisée d'une cible (sentiments nationalistes, jugements tranchés, formules caustiques, sens tactique...). Les "aspects» des individus sur lesquels se portent préférentiellement les jugements sont les opinions $(26 \%)$, les traits de caractère $(26 \%)$, les actions $(17 \%)$, les attitudes sociales $(15 \%)$, les compétences $(10 \%)$ et les propos $(4 \%)$. Les expressions de chaque catégorie sont à leur tour finement analysées. Prenons le cas des opinions. Quand un jugement prend pour objet les opinions d'un individu, les qualificatifs se rattachent à des termes tels que positions, sympathies, engagements, opinions, idées, thèses, convictions, options, vues, sentiments. Parmi nos observables, ces 10 substantifs ramènent 67 attestations, c'està-dire $60 \%$ des jugements classés dans cette catégorie. La forme positions est présente dans 26 attestations, où elle cooccure 13 fois avec l'adjectif conservatrices. Les qualités attribuées à ces cibles appartiennent principalement aux domaines politique et idéologique (positions xénophobes, convictions démocratiques, engagements nationalistes, idées pacifistes, opinions conservatrices, vues progressistes, sympathies progressistes, jugements tranchés, options jacobines, sentiments libéraux). Ce qui est également le cas des termes simples comme pacifisme, humanisme, xénophobie, francophilie, libéralisme, eurosceptisme.

Cette construction, très faiblement modalisée, sert essentiellement à exprimer des jugements assurés, où l'attribution de la qualité est catégorique, ne comportant ni condition, ni alternative, ni restriction temporelle. Elle est donc parfaitement adaptée à l'expression de la réputation.

L'influence du corpus est évidente. La part élevée des cibles humaines visées par la construction en est la première conséquence. Les détenteurs des opinions soumises à des appréciations sont des acteurs de la vie politique et sociale. Parmi les actions et les réalisations invoquées, ce sont des réalisations artistiques qui prédominent.

\subsection{Etude de constructions polysémiques}

Nous avons réalisé une étude approfondie sur le patron $\mathrm{P} 17$ «en $\mathrm{N}+<\exp >$ » dans une réalisation particulière : "ponct En|en (très) adj $\operatorname{mot}\{1,15\}$ ponct 》 avec adj $\rightarrow$ bon $\mid$ vrai $\mid$ digne $\mid$ authentique $\mid$ véritable $\mid$ simple $\mid$ vieille $\mid$ vieux $\mid$ jeune $(+$ variations morphologiques). Cette configuration, même construite sur un sousensemble restreint et homogène d'adjectifs enclosures ${ }^{12}$, reste ambiguë.

12. Termes qui modalisent (le degré de) l'appartenance d'une entité à une catégorie. 
Sur un corpus formé de quatre ans $d u$ Monde $^{13}$, toute catégorie confondue ${ }^{14}$, 398 attestations ont été trouvées. La construction n'est pas dédiée à l'évaluation des sujets humains, mais cet emploi reste majoritaire dans notre corpus : $59 \%$ des attestations prennent pour cible des individus ou des organisations. Toutefois, les expressions qui apportent du bruit ne sont pas nombreuses et de ce fait peuvent facilement être écartées (* en bonne logique, en bonne voie, en bonne santé, en bonne partie, en bonne et due forme...).

Le deuxième constat met en lumière une très inégale représentation de ce patron selon les adjectifs (bon : 174 ; vrai : 18 ; véritable : 13 ; vieux : 13 ; digne : 10 ; simple: 3 ; authentique: 2 , jeune $: 1)$. Ce résultat mérite d'être vérifié sur d'autres corpus.

Quelles valeurs peut prendre l'expression « en bon $\mathrm{N}$ » quand $\mathrm{N}$ renvoie à un humain ? L'observation des champs lexicaux couverts par les substantifs en question, corrélée avec l'examen des prédications principales, met en lumière un résultat notable. Sur 173 attestations, 57\% des emplois dénotent l'idée de conformité (ou de conformisme), $32 \%$ expriment l'appréciation d'une capacité ou d'une aptitude, dans $12 \%$ des cas ces deux valeurs sont cumulées. Le jugement de conformité renvoie à l'attitude de quelqu'un qui règle ses idées, son comportement, sur ceux de son milieu (religieux, politique, social, culturel, familial) : en bon fils de la bourgeoisie brestoise, en bon Alsacien, en bon spinoziste... (9). Dans la terminologie Appraisal, il s'agit de la catégorie «Gradation » (focus renforcé). Le jugement de qualité quant à lui correspond à un jugement positif porté sur des capacités (savoir-faire, habileté) manifestées par une personne : en bon contrebassiste, en bon avocat, en bon professionnel... (10). Dans la terminologie Appraisal, il s'agit de la catégorie « Attitude » (jugement > capacité).

(9) En bon citoyen helvétique, H. K. pourrait passer pour un compositeur $d u$ juste milieu, entre l'appel de l'inouï façon Stockhausen et le recyclage des repères à la Ligeti.

(10) En bon feuilletoniste, H. J. conduit judicieusement - et même poétiquement - son intrigue vers un final spectaculaire...

\section{Patrons linguistiques et facettes de l'évaluation}

Nous allons maintenant examiner les différentes expressions axiologiques attestées en position détachée d'un point de vue sémantique et fonctionnel. Que nous apprennent-elles sur l'acte d'évaluation? Pour répondre à cette question, nous

13. Quatre ans du Monde (2003-2006) : 1 Go (environ 170 millions de mots).

14. Ici, le choix d'étendre la gamme des articles concernés (au-delà des portraits et des biographies) se justifie par la nature de la construction laquelle cible l'actant dans sa qualité d'agent. Ce sont donc a priori des énoncés d'action que nous visons. 
procéderons en deux temps. Nous nous intéressons tout d'abord à la sémantique « locale» des constituants détachés. Parmi les structures évaluatives compatibles avec le détachement, certaines se spécialisent pour encoder des aspects précis de l'acte d'évaluation (sections 4.1-4.5). Ensuite, dans la section 5, nous traitons d'une part, des rapports de sens entre constituants détachés et la prédication principale, et, d'autre part, des relations sémantiques entre constituants détachés multiples.

Dans les deux cas, nous analysons les différents patrons dans leur expression minimale et avec des expansions. En effet, les segments périphériques à caractère axiologique, bien que synthétiques par nature, peuvent voir s'adjoindre des éléments (modificateurs adverbiaux, connecteurs...) capables d'orienter l'interprétation de l'acte d'évaluation (végétarienne vs. végétarienne affichée).

\subsection{Portée du jugement}

La portée d'un jugement d'évaluation touche au délicat problème de savoir si la qualité attribuée à une cible se manifeste de manière durable ou permanente (qualité pouvant être vue comme inhérente ou essentielle) ou seulement de manière circonstanciée, en situation (qualité accidentelle) ?

Dans (11), aucun intervalle temporel, aucune circonstance particulière ne limitent l'application à la personne concernée des différentes caractéristiques énumérées (méthodique, rationnel). Dans (12), en revanche, la caractéristique en bon soldat ne s'applique à la personne concernée qu'en tant que l'agent de l'action appliquer la politique...

(11) Méthodique, rationnel, il aime la gestion quotidienne de l'entreprise, le métier, mais n'entend rien à la stratégie et au long terme.

(12) Ils savent que, en bon soldat, R.D. applique la politique voulue par son mentor qui ne peut la lâcher sans se désavouer.

Principalement, trois patrons permettent d'encoder une liaison essentielle ou durable de l'individu avec la qualité dénotée. Tout d'abord, P1 « homme/femme de $\mathrm{N} »$, incompatible avec des prédicats indiquant une extension temporelle (13). Ensuite, le patron P5, correspondant à des syntagmes nominaux sans déterminant de nature métaphorique (virtuose $d u$ suspense, champion des ennuis judiciaires...) (14), tout aussi récalcitrants à des restrictions temporelles. Notons enfin des tours tels que connu pour, réputé pour, couronné de lauriers... (P16) qui présentent explicitement la qualité dénotée par le segment détaché comme faisant partie d'une réputation (15).

(13) Femme de caractère, cette passionaria de la pub cumule les titres chez Havas.

(14) Le prestigieux prix Nobel a été décerné au populaire romancier français, magicien de la langue. 
(15) Mme T., connue pour sa personnalité énergique et flamboyante, avait déjà été Premier ministre - première femme à occuper ce poste en Ukraine - de février à septembre 2005 (...).

Dans des constructions moins spécialisées, on note l'influence possible de certaines marques contextuelles (par nature, d'ordinaire, comme à son habitude, notoire, notoirement...). Leur rôle est de présenter la qualité comme inscrite dans le caractère même de l'individu ou comme appartenant à son comportement habituel (16-17).

(16) Anti-diva par nature, elle récolte les fruits de sa très grande discrétion.

(17) L'alliance obligée qu'il a nouée avec la Ligue du Nord, eurosceptique notoire, ajoute au malaise ambiant.

Une qualité peut être associée contextuellement à un individu en situation, c'està-dire, prédiquée de lui en tant qu'il est actant d'une action (agent, plus rarement patient). Plusieurs types de constructions détachées, par exemple de nature adverbiale (adverbiaux de manière orientés sujet, des adverbiaux d'attitude...), de par leur propriété de porter sur l'ensemble de la relation prédicative, permettent de dénoter de telles qualifications « contextualisées ».

C'est le cas de la construction P17 : en $\mathrm{N}<\exp >$, telle que en $N$ ou en adj $N . .$. (en visionnaire, en bon adepte de..., en digne épicurien) avec un vaste paradigme de noms possibles (métiers, fonctions...). Elle se laisse paraphraser en termes causaux (voir section 5.1).

(18) En digne émule des entreprises privées, le groupe vient de créer une direction du développement.

Notons aussi le tour « en tout/toute $\mathrm{N} »$ (en toute clarté, en toute cordialité, en toute discrétion...) tel qu'exemplifié dans (19).

(19) $<$ titre> H. chez S., en toute discrétion.

Enfin, il est possible de marquer explicitement que la qualité en question se déploie préférentiellement ou exclusivement pour certains types de tâches (20). Cette forme de restriction est encodée par des expressions correspondant au patron (P12) :

(20) Derrière l'exubérance se profile cependant un chef d'entreprise avisé, fort habile pour vendre et exporter ses spectacles, devenus de grosses productions.

L'impossibilité de signaler qu'un jugement d'évaluation ne concerne l'individu que dans certaines circonstances précises constitue, à nos yeux, une limite importante du modèle Appraisal.

\subsection{Cible}

Un jugement d'évaluation peut porter sur une cible dans sa globalité ou bien se focaliser sur certains de ses aspects (trait de caractère, qualité du tempérament, 
talent, qualité relationnelle, compétence, manière d'agir ou de se comporter..., s'il s'agit de personnes).

Les constructions qui rendent compte d'un regard global (direct) sur un individu sont celles qui n'induisent pas de spécification ou de partition. C'est le cas des patrons P1 et P11 (21).

(21) Homme de compromis, modeste et chaleureux, JL avait conquis l'amitié du président Vaclav Havel qui lui a rendu un vibrant hommage.

Les moyens d'expression permettant un regard analytique sont bien plus nombreux. Les constructions absolues (P4) en sont l'exemple le plus parlant. «Les constructions absolues, par le rapport partie/tout qu'elles impliquent d'ordinaire, ne qualifient pas directement le référent, mais un de ses aspects ou une réalité qui se trouve en relation avec lui. » (Combettes, 1998, p. 21). Quand le référent est de type humain, ces constructions font appel à des champs lexicaux spécifiques : (i) parties $\mathrm{du}$ corps qui participent à la communication (yeux, visage, oreilles...) ou qui symbolisent certaines qualités relationnelles (cœur, geste...), (ii) certaines facultés intellectuelles (écoute, regard...) ou «produits» abstraits de ces facultés (idées) (22-23), (iii) enfin, des éléments de l'apparence (tenues...) peuvent également y être mentionnés. Multipliée, cette forme d'appréciation focalisée sert notamment à brosser des portraits de personnes ou d'institutions.

(22) Riche en idées, mais endetté, il a tout d'un meneur d'hommes, main tendue et grand cour, poigne ferme et aeil avisé.

(23) Ses débuts fracassants et sulfureux ont joué un rôle non négligeable dans l'édification de la légende du Robin des bois du web, cheveux longs, idées libertaires et tenues brouillonnes.

\subsection{Polarité}

La question de la polarité des mots, des expressions ou des énoncés est, on le sait, particulièrement complexe. On trouvera dans (Kerbrat-Orecchioni, 2002) un exposé très éclairant sur la diversité des facteurs qui influent sur l'interprétation axiologique des jugements.

Dans la perspective qui est la nôtre, il est utile de savoir si certaines constructions privilégient une polarité plutôt que l'autre. Nous avons traité cette question pour les patrons $\mathrm{P} 1, \mathrm{P} 11$ et $\mathrm{P} 16$. Mais, nous avons réussi à y répondre seulement relativement à la construction "homme|femme de $\mathrm{N} »$. Dans le corpus exploré (16 ans du Monde), elle renvoie dans environ $85 \%$ des cas à des jugements positifs. Minimale et synthétique, la structure $\mathrm{P} 1$ semble « sélectionner » ses qualités à un certain niveau de généralité (fidélité, courage, droiture, sincérité, dévouement, modération, rigueur...). Par conséquent, son interprétation axiologique est relativement aisée. En 
revanche, les deux autres patrons renvoient à des structures formellement plus complexes et sémantiquement plus riches.

(24) Femme de tête, femme de tripes, femme d'influence et femme d'ambition, $L$. R. assume le deuil d'une nation...

Le groupe adjectival (P11) permet d'exprimer des jugements nuancés ou contrastés, notamment au moyen de termes connectés entre eux (par mais, et, plus... $q u e, n i \ldots n i \ldots$ ). Dans ce cas, le calcul de la polarité du groupe entier devient très vite non trivial (25-26). On retiendra que les qualités ainsi mises en relation sont sémantiquement similaires ${ }^{15}$, propriété fort utile pour une collecte automatique d'adjectifs axiologiques.

(25) Militant mais opportuniste, franc-tireur mais habile, sociable mais anticonformiste, le directeur de l'Opéra de Paris sait manier les paradoxes pour parvenir à ses fins.

(26) Ni régionaliste, ni cosmopolite, il capte l'air du temps et synthétise.

Le tour P16 sélectionne des aspects particuliers de la cible sur lesquels s'est focalisé le jugement (section 3.2). Il s'agit souvent de qualités très fortement spécifiées, notamment par renvoi à des univers de référence. Dans notre corpus, près de $30 \%$ d'attestations renvoient à des opinions et des propos (connu pour ses positions lä̈ques ouvertes... ses vues planificatrices et centralisatrices... ses options jacobines... sa rigueur idéologique communiste... son eurosceptisme...). L'évaluation axiologique de ces objets ne peut qu'être relative (27).

(27) Né en 1914, C. B., qui fut le dernier premier ministre du chah d'Iran, avait été un grand bourgeois anti-clérical, et le fidèle héritier de Mossadegh, l'homme qui nationalisa le pétrole iranien et qui fut au pouvoir de 1951 à 1953. Bien qu'appartenant à la tribu des Bakhtiar, réputée pour sa fidélité au chah, il n'avait jamais dissimulé son hostilité au souverain et à son entourage. Peut-être devait-il son attachement aux idéaux républicains au fait qu'il avait fait ses études secondaires et universitaires en France.

\subsection{Intensité}

L'appréciation de la force ou de l'intensité d'une qualité est un élément essentiel dans un acte évaluatif. Certaines constructions permettent d'exprimer des cas

15. « On ne coordonne bien (on veut dire de façon naturelle, sans effet d'ironie ou de rupture) que des termes qui sont sémantiquement à peu près assortis » $(. .$.$) «Il n'en reste pas moins$ que cette question de la compatibilité sémantique qui rend compte de l'impossibilité, souvent signalée de coordonner un adjectif qualificatif à un AR (adjectif de relation) » (Noailly, 1999, p. 75). Exemple : *une politique familiale et audacieuse. 
remarquables : (i) le haut degré de réalisation d'une qualité, (ii) l'écart par rapport à une norme, à ce qui est attendu, (iii) une manifestation nuancée.

Le génitif de qualité (P13) construit sur le schéma: (\{doté, doué...\}) de Art_indéf (Adj) N (Adj), permet de rendre compte d'une réalisation remarquable (exceptionnelle, extrême, remarquable, extrême, absolue...) d'une qualité (d'une détermination farouche, d'une extrême fermeté, d'une patience exemplaire, d'une rigueur absolue... (28-29).

(28) Pur produit du sérail, l'inspecteur des finances est le parfait modèle de "l'honnête homme » cher à l'Hexagone : d'une courtoisie extrême, il est aussi à l'aise pour parler de finance internationale que de l'œuvre d'Alain RobbeGrillet, dont il fut un proche.

(29) Dotée d'un coup d'oeil remarquable, elle n'a pas son pareil pour sélectionner les bonnes peaux et les négocier au meilleur prix.

Dans des constructions de nature adverbiale (P18), des classificateurs tels que une dose de, un brin de, une pincée de permettent au contraire d'apprécier finement les différentes manières d'agir (non sans un brin de machiavélisme, ...) (30). Ce type de modalisateurs vient atténuer le caractère parfois un peu brutal ou direct des jugements évaluatifs.

(30) Avec un brin de provocation, $F$. A. retourne la question à ses interlocuteurs : que font-ils pour faire réussir les autres?

D'autres constructions, bâties sur le groupe adjectival (P11), mettent en avant une appréciation relative qui traduit des écarts par rapport à une norme (à ce qui est attendu) : pas assez politique, trop sociétale, trop puissant pour être raisonnable; trop policé pour être authentique; trop naif pour savoir combattre; trop raffiné pour être compris par d'autres hommes... C'est un jugement de "normalité» (au sens d'Appraisal), relativement à la nature ou la situation de la cible (équilibre avec d'autres caractéristiques...) (31).

(31) N. est riche, élégante... et un peu excentrique pour une Rothschild.

Signalons enfin les expressions (P20) qui fournissent une appréciation de la « situation» d'un individu, en le situant abstraitement à différentes positions (haut, bas, sommet, creux...) dans un espace symbolique orienté, représentant selon les cas son parcours, ses performances, sa popularité, etc. (32).

(32) Au plus bas dans les sondages, le sévère Premier ministre britannique a pour une fois trouvé dans l'humour - "la politesse du désespoir » comme on dit - une manière de solliciter un peu d'indulgence auprès de ses troupes. 


\subsection{Engagement, authenticité}

Intéressons-nous maintenant à des configurations où l'évaluation appelle un jeu de positionnements intersubjectif. Le premier cas est celui où l'énonciateur indique qu'il n'est pas la source du jugement en question, sans avoir nécessairement à prendre position par rapport à la justesse de ce jugement. Rapporter une appréciation produite par un tiers (33), préciser que l'appréciation rapportée est une auto-appréciation (34), signaler qu'une qualité est délibérément mise en avant par «sa » cible (35) sont quelques stratégies qui minimisent la responsabilité de l'acte évaluatif.

(33) Perçu comme un sauveur et considéré par les anciens d'Aérospatiale comme l'homme providentiel, certains commencent néanmoins à s'interroger sur la méthode Gallois.

(34) Doté selon ses propres propos "d'une grande capacité d'écoute », il veux que le CSA soit « le garant de la liberté... ».

(35) Végétarienne affichée, elle défend les animaux, sauf s'ils sont politiques.

Le deuxième cas de figure est celui où l'énonciateur produit une appréciation sur l'authenticité d'une qualité attribuée à quelqu'un ou manifestée par lui. Il peut signaler explicitement le caractère plus ou moins superficiel, feint ou trompeur (ce timide déguisé en austère, pseudo-philosophe autodidacte, faussement neutre...) d'une attitude ou d'un trait de caractère (36). Remarquer une (fausse) ressemblance avec quelqu'un de connu ou dénoncer une imitation plus ou moins heureuse d'une personnalité publique sont des modalités possibles d'une telle attitude (37).

(36) «La propriété est maintenant estimée à 120 millions d'euros », glisse, faussement modeste, B.M.

(37) ... de la plume d'un petit homme de 81 ans, aux faux airs de Woody Allen.

Inversement, souligner que $\mathrm{X}$ est authentiquement européen ou que $\mathrm{X}$ a accompli quelque chose avec une authentique conviction ou avec une modestie non feinte sert selon les cas (i) à éloigner un doute possible sur la réalité de la qualité en question, et/ou (ii) à mettre en lumière favorable son caractère précieux ou rare (38-39).

(38) Avec une authentique conviction, il a confié sa foi dans les grands magasins et livré sa vision de l'avenir du groupe.

(39) Avec une modestie non feinte, emprunte de l'élégance...

Enfin, mentionnons des expressions telles que «vrai N/adj et faux N/adj» permettant d'associer ce qui est trompeur (faux...) dans l'apparence de quelqu'un avec une autre qualité jugée, elle, bien réelle (40-41). Elles méritent, du fait du présupposé dont elles sont chargées, une attention particulière et une étude approfondie. La question des polarités dans cette structure coordonnée (d'apparence) bipolaire reste également à approfondir pour expliquer le mécanisme qui, au final, fait converger les deux jugements qui la composent. 
(40) Car la gageure est bien là pour ce défenseur de la liberté d'expression, vrai rebelle et faux intrépide : trouver un partenaire qui accepte ses conditions...

(41) < Titre> : La mort de J.V., faux naïf et vrai comédien.

\section{Relations sémantiques au sein des énoncés à constituants détachés}

Constituant du discours d'apparence libre, le segment détaché entretient avec le reste de l'énoncé, qu'il s'agisse de la prédication principale ou d'autres segments détachés, des rapports de cohésion. L'absence de dépendances syntaxiques n'exclut pas l'existence de liens sémantiques clairement identifiables. Nous nous attacherons à isoler des schémas réguliers qui mettent en jeu patrons et certaines catégories de marques discursives.

\subsection{Rapports entre le CD et le noyau prédicatif de la phrase}

Intéresserons-nous tout d'abord à la nature des relations sémantiques qui peuvent s'établir entre un constituant détaché de nature axiologique et la prédication principale. Cela revient à s'interroger sur les fonctions possibles d'un acte évaluatif. Dans les énoncés que nous avons analysés, trois relations se détachent : l'opposition, la causalité et l'élaboration.

Les énoncés évaluatifs à constituants détachés se prêtent aisément à l'expression des oppositions, des contrastes ou des contradictions. Quand le segment extraprédicatif dénote une qualité attribuée à une personne, ce type de rapport peut traduire selon les cas : (i) un contraste entre deux caractéristiques concomitantes de la personne, (ii) une contradiction entre la réputation de cette personne (qualité qui lui est socialement reconnue ou perçue comme durable) et son comportement (action, discours...) du moment; (iii) une évolution dans le temps (hier/ aujourd'hui...) conduisant à une inversion possible de la polarité du jugement.

L'opposition peut être exprimée par le lexique : paires de termes antithétiques (audacieux - timidité, calme - taraudé par le doute, couronné - sanctionné), des indices grammaticaux (pourtant, néanmoins, pour autant...), certaines configurations spécifiques (aussi ... soit il/elle, X être avant tout...).

(42) Aussi lettrée et séduisante soit-elle, L.P. est avant tout une héritière.

(43) Adepte d'une politique tarifaire volontaire mais audacieuse, G.M., qui promène la réputation d'un directeur dispendieux, n'a pas pour autant laissé filer les comptes.

(44) Et le commissaire extraordinaire nommé par le gouvernement le 11 janvier, G.DG., pourtant réputé pour être un homme à poigne, ne parvient pas à ... 
(45) Couvert d'honneurs, l'homme L-S. avoue pourtant avoir du mal à aimer son époque.

(46) Perçu comme un sauveur et considéré par les anciens d'Aérospatiale comme l'homme providentiel, certains commencent néanmoins à s'interroger sur la méthode Gallois.

(47) Audacieux pour mener des réformes, dont la liste est impressionnante, habile à construire du neuf en matière sociale, le chef de l'Etat montre une timidité certaine dans la lutte contre les déficits.

Une qualité dénotée par un segment détaché peut être présentée comme la cause d'une situation (visée ou non), décrite dans la proposition principale. Ceci permet par exemple d'expliquer la réussite ou l'échec d'une action par une qualité (capacité, ténacité...) de celui qui l'accomplit. Les marques causales explicites (P19 : grâce à, à force de...) et des adverbes orientés vers le sujet (P18 : courageusement...) jouent, dans le contexte qui est le nôtre, un rôle analogue. En effet, pour ce qui concerne ces derniers : «Les adverbes de sujet-phrase orienté vers le sujet évoquent une cause émanant de l'agent; cette cause est une propriété contingente de l'agent, une propriété qui ne lui appartient que dans la situation qui le conduit à mettre en œuvre le procès signifié par le prédicat. (...) L'adverbe de sujet-phrase est incompatible avec toute forme verbale à valeur générique, le présent de narration p.ex.)» (Guimier, 1996, 89).

Des constructions détachées de type en $N$, en adj $N$ (P17) appellent également une interprétation causale. Franckel et Lebaud (1991, p.71) expliquent leur fonctionnement, (sur l'exemple de En homme avisé, il a réservé sa réponse), de la manière suivante : "En 'homme avisé', s'interprète comme une forme de repère causal: sa qualité d'homme avisé l'a fait agir comme il a agi (en réservant sa réponse). Une glose de l'énoncé pourrait être : c'est un homme avisé et, de ce fait, il a réservé sa réponse ». Cette analyse est également confirmée par (Vigier, 2008).

Notons l'influence des modalités d'action telles que réussir à, parvenir à... qui contribuent à une lecture intentionnelle des énoncés (causalité volitive, au sens de la RST).

(48) Grâce à son travail et à sa ténacité, P.B. a su créer aujourd'hui une entreprise à l'avant-garde du Net.

(49) Avec persévérance et beaucoup de diplomatie, Y.N. a finalement réussi à se faire accepter des 300 Bréhatins qui vivent sur l'île toute l'année.

(50) Toutes les marques-enseignes n'ont pas le statut requis pour cela, mais, à force de constance et de volonté, certaines y sont parvenues.

(51) En véritable requin de studio, il a accompagné tout au long des années 70 et 80 bon nombre de chanteurs français influencés par les musiques d'OutreAtlantique... 
La relation d'élaboration ${ }^{16}$, quant à elle, intervient généralement dans des énoncés, dont la prédication principale sert elle aussi à exprimer un jugement (5253). Les rapports d'élaboration traduisent, par exemple, le fait que telle facette de personnalité illustre ou confirme tel profil général, que tel comportement particulier s'accorde avec telle manière d'agir habituelle. Les constructions absolues (P4) se prêtent donc parfaitement à ce type d'emploi.

(52) Riche en idées, mais endetté, il a tout d'un meneur d'hommes, main tendue et grand cour, poigne ferme et ail avisé.

(53) Bouillonnant d'idées, cet hyperactif compense son manque de créativité par un sens aigu de la formule.

Pour systématiser, ces relations rhétoriques peuvent être signalées de différentes manières : (a) par la structure même du patron (P4 : relation d'élaboration), (b) par un élément constitutif du patron (P19 : à force de $\mathrm{N}$ : relation de causalité), (c) par le choix du lexique saturant le patron (P11 : paires d'adjectifs à la polarité opposée, ex. audacieux - timide: relation d'opposition), (d) enfin, par des connecteurs argumentatifs (mais, pourtant : opposition).

\subsection{Rapports sémantiques entre CD multiples}

Dans les énoncés à constituants détachés multiples, on rencontre des jugements analytiques mettant en lumière plusieurs qualités mises en relation les unes avec les autres. Il peut s'agir du point de vue temporel, de la simple co-coexistence, de l'alternance ou de la succession entre deux qualités. Co-présentes, ces qualités peuvent se renforcer ou s'opposer.

Ces relations peuvent être exprimées de différentes manières. La coexistence de deux qualités au même titre peut être signalée par la structure coordonnée de type «mi-qual1, $m i$-qual2 » qui introduit pour un référent une double qualification (54). Conjointement présentes, ces qualités peuvent être antinomiques (mi-réel, mivirtuel).

(54) Gueule sympathique, mi-séducteur, mi-confesseur : c'est un Français comme on les aime.

Notons ensuite la possibilité de rendre compte par le biais du détachement de la succession temporelle de plusieurs états qu'a vécus un individu (55) ou de l'alternance de qualités ou d'états (56) (tantôt... tantôt).

16. La relation d'élaboration, au sens de la RST, s'établit entre deux segments N (noyau) et S (satellite) par le fait que « $\mathrm{S}$ introduit un détail supplémentaire sur la situation ou un élément décrit dans $\mathrm{N} »$, sur la base de rapports tels que: ensemble/composant, abstraction/concrétisation, tout/partie, processus/étape, objet/attribut ou généralisation/détail. 
(55) Donnée pour morte avant-hier, pratiquement prête à fondre en larmes hier matin, H.C. avait retrouvé le sourire hier soir.

(56) Ce veilleur, tantôt candide, tantôt désabusé, est une sorte de marginal, inapte à affronter la jungle moderne.

L'opposition entre plusieurs qualités attribuées au même individu s'exprime le plus souvent par des connecteurs explicites (mais qui, mais dont...) (57) ou encore des paires lexicales antinomiques (méconnu/célèbre) (58).

(57) Il publie son autobiographie «Dreams of My Father », histoire d'un enfant noir dans un monde de blancs, en quête de son père, économiste brillant, mais qui finit sa vie alcoolique et se tue dans un accident de voiture.

(58) Méconnu en France, célèbre aux Etats-Unis, A.S. est un grand $d u$ photojournalisme.

Un renforcement (explicité par voire, et même...) entre deux qualités montre au contraire qu'elles vont dans le même sens du point de vue de la polarité du jugement (59).

(59) Discret, voire solitaire, réputé pour son sang froid, il n'a pas été surnommé pour rien "le Boa» après avoir avalé le Crédit Lyonnais et son amour de la corrida ne l'a pas transformé en victime consentante.

\section{Synthèse}

$\mathrm{Au}$ terme de ce travail, nous arrivons à une somme de résultats de portée et d'importance variables. Ces résultats proviennent de plusieurs études exploratoires, sur des corpus très ciblés et restreints, ne relevant pas d'une démarche nécessairement unifiée. Il y a, tout d'abord, un ensemble de patrons correspondant à des formes récurrentes, potentiellement porteuses de contenus évaluatifs en apposition. Ensuite, issues de l'observation des attestations de ces patrons sur corpus, (i) proposition de paramètres ou de valeurs saillantes de paramètre caractéristiques de l'acte évaluatif, (ii) quelques indications tendancielles sur la sémantique des qualités associées à des personnes, (iii) observations et critiques portées sur le modèle Appraisal. Les patrons ne sont donc pas une fin, mais un moyen permettant de progresser dans la caractérisation linguistique de l'acte d'évaluation.

Nous avons montré que parmi les constructions employées en apposition certaines se spécialisent (ou sont employées préférentiellement) pour dénoter des aspects particuliers de l'acte d'évaluation (tableau 3).

Ces informations traduisent des préoccupations et des visées pragmatiques de ceux qui émettent des jugements évaluatifs. Les qualités attribuées à autrui sont-elles authentiques (AUTHENTICITE) ? Le caractérisent-elles de manière essentielle, durable ou seulement passagère voire accidentelle (INHERENCE) ? Ciblent-elles sa personnalité dans son ensemble ? ou uniquement certains de ses traits (ANGLE 
D'APPROCHE) ? Se manifestent-elles en deçà ou au-delà de ce qui est attendu ou convenable (CONFORMITE) ? Enfin, sont elles chargées axiologiquement de manière stable (universelle) ou seulement de manière relative (RELATIVITE) ? Ces différents paramètres, dont la désignation n'est pas encore stabilisée, viendront enrichir le futur modèle de l'évaluation.

\begin{tabular}{|l|l|c|}
\hline Paramètre & \multicolumn{1}{|c|}{ Valeurs } & $\begin{array}{c}\text { Patrons concernés en } \\
\text { priorité }\end{array}$ \\
\hline $\begin{array}{l}\text { RELATIVITE } \\
<\text { Axiologie }>\end{array}$ & $\begin{array}{l}\text { Axiologie relative (instable) } v s \\
\text { universelle (stable) }\end{array}$ & tous \\
\hline $\begin{array}{l}\text { ANGLE D'APPROCHE } \\
<\text { Cible }>\end{array}$ & $\begin{array}{l}\text { Approche totalisante (globale) } v s . \\
\text { focalisante (partielle) }\end{array}$ & P1-P4, P11, P13, P14 \\
\hline INHÉRENCE $<$ Qualité $>$ & $\begin{array}{l}\text { Qualité inhérente (permanente, } \\
\text { durable) vs. contingente } \\
\text { (accidentelle) }\end{array}$ & P1, P5, P16, P17-P20 \\
\hline $\begin{array}{l}\text { AUTHENTICITÉ } \\
<\text { Qualité> }\end{array}$ & $\begin{array}{l}\text { Qualité authentique (naturelle) } v s . \\
\text { feinte (apparente) }\end{array}$ & P11, P18 \\
\hline $\begin{array}{l}\text { CONFORMITE } \\
<\text { Qualité }>\end{array}$ & $\begin{array}{l}\text { Qualité (ou intensité de la } \\
\text { qualité) conforme (attendue) } v s . \\
\text { non conforme (imprévue) }\end{array}$ & P11, P16, P18 \\
\hline
\end{tabular}

Tableau 3. Quelques paramètres de l'évaluation

Le deuxième point que nous souhaitons souligner est le rapport privilégié entre l'évaluation et d'autres opérations ou relations langagières. Dans la section 5, nous avons vu qu'un attribut doté d'une charge axiologique est invoqué lorsqu'il est en relation (explicative, illustrative, contrastive...) suffisante avec le propos tenu sur la cible. L'étude fait ressortir également un lien significatif entre l'évaluation et la catégorisation. Du point de vue des opérations ou des relations linguistiques encodées, il est possible de distinguer : (i) des patrons qui renvoient à la qualification seule ( $\mathrm{P}$, P11, P18) courageux, avec pragmatisme... ; (ii) des patrons qui associent qualification et catégorisation ${ }^{17}$ (P1, P2, P6, P17...) : femme de caractère, économiste brillant, patron respecté, en politicien expérimenté...; (iii) des patrons qui associent qualification et prise en charge (P16) connu pour son intégrité... (iv) des patrons qui s'appuient sur une relation partie-tout implicite (P4, P14...) : ceil avisé, d'un bras vigoureux...; (v) des patrons qui encodent une relation causale (P17, P19) : à force d'obstination, en musicien de talent... ; (vi) des patrons qui dénotent une localisation abstraite (P20) au creux de la vague, au sommet de son art...

17. L'appartenance catégorielle (sociale, professionnelle...) des entités cibles d'évaluation, laquelle influence et oriente les jugements de valeur, reste une question à approfondir. Nous songeons à une recherche sur corpus qui permettrait de mettre en évidence des collocations marquant des rapports privilégiés entre types de cible et types de qualité. 


\section{Pour conclure}

Très présente en TAL ces dernières années, notamment dans la recherche informatisée des opinions, des attitudes ou des émotions dans des documents textuels, la notion de jugement d'évaluation n'a pas reçu en linguistique française de description suffisante pour donner un appui solide à ces applications finalisées.

L'étude que nous venons de présenter contribue à la modélisation fine du phénomène évaluatif dans la langue et à la construction de ressources linguistiques exploitables par des applications informatiques. Nous nous sommes concentrée sur l'analyse sémantique de certaines structures textuelles, dont les caractéristiques formelles et sémantiques nous paraissent particulièrement intéressantes dans cette optique.

Le système appositif permet de faire mention des attributs saillants ou socialement valorisés des individus et de les présenter comme relativement consensuels. De ce point de vue, nos résultats peuvent intéresser des applications sur la réputation, des recherches sur des catégories socio-professionnelles et leur visibilité dans les médias, etc.

Il est légitime de s'interroger sur la part que représentent les énoncés à constituants détachés axiologiques dans des textes («tout venant», journalistiques, romans, récits de vie, nécrologies...). Aujourd'hui, nous ne sommes pas en mesure d'y répondre. Nous ne disposons pas d'étude de grande ampleur sur l'emploi du système appositif dans différents genres de discours ou types de textes ${ }^{18}$. Savoir en apprécier la nature (axiologique ou non) relève d'un défi plus important encore, car cela nécessite le concours d'un interprétant humain.

Au stade actuel, l'apport essentiel de ce travail est d'avoir montré que certaines structures dénotent des caractéristiques significatives des actes évaluatifs et à ce titre elles pourront être exploitées, avec les amorces de lexiques constitués, dans des traitements informatiques. Nous avons également entamé un examen critique du modèle Appraisal qui sera poursuivi selon la même méthode.

18. Il existe en revanche des études très ciblées permettant de mesurer la difficulté d'une telle entreprise. On trouve dans (Neveu 1998, 166-167) des données statistiques sur l'emploi du système appositif (nominal et adjectival, sans contrainte sémantique) dans un corpus homogène, constitué de quatre romans (auto)biographiques de J.-P. Sartre. La densité des occurrences (1050 pour 500 pages) est jugée importante par F. Neveu. En même temps, un fort déséquilibre dans la distribution de ces occurrences selon les textes est constaté. Notons que dans (Jackiewicz et al., 2009a), nous avons obtenu un résultat comparable. Une répartition clairement hétérogène des 10 patrons analysés, à l'intérieur d'un corpus très homogène composé d'articles de type portrait ou biographie tirés du Monde, a été mise en évidence. Ainsi qu'une forte concentration de patrons au sein de quelques articles. L'existence de modes préférentiels d'expression selon les auteurs est donc un facteur significatif, mais, comme le montre l'étude de Neveu, non suffisant. 
Les recherches que nous venons de présenter sont focalisées sur le détachement. Toutefois, nos observables permettent de constater que plusieurs constructions qui fonctionnent en position détachée peuvent occuper également des places argumentales dans la prédication principale (60-62). C'est un des prolongements logiques du travail décrit ici.

(60) Piètre orateur, l'austère et cérébral G.B. est notoirement mal à l'aise dans ce genre d'exercice.

(61) Ainsi, à mi-mandat, cet apôtre de la modernité, accusé par la presse d'être le fossoyeur de l'Opéra, se révèle plus pragmatique qu'il n'y paraît.

(62) Nommé à la tête de la Réserve fédérale en 1970, A.B. avait été le dandy de l'inflation.

\section{Remerciements}

Je tiens à remercier les experts anonymes pour la relecture minutieuse de ce texte, ainsi que pour leurs conseils fort pertinents.

\section{Bibliographie}

Anscombre J.-C., «Temps, aspect et agentivité dans le domaine des adjectives psychologiques », Revue Lidil, $\mathrm{n}^{\circ}$ 32, 2005.

Bednarek M., "Language patterns and ATTITUDE”, Functions of Language 16:2, John Benjamins Publishing Compagny.

Bloom K., Garg N., Argamon S., "Extracting Appraisal Expressions", HLT/NAACL 2007, $2007 \mathrm{a}$.

Bloom K., Stein N., Argamon S., "Appraisal Extraction for News Opinion Analysis", NTCIR6 2007, 2007b.

Buvet P.-A., Girardin C., Gross G., Groud C., « Les prédicats d'affects », Revue Lidil n ${ }^{\circ} 32$, 2005.

Charaudeau P., La grammaire du sens et de l'expression, Paris, Hachette Education, 1992.

Combettes B., Expressions détachées en français, Ophrys, Collection L'essentiel Français, 1998.

Esuli A., Sebastiani F., «Sentiwordnet: A publicly available lexical resource for opinion mining », Proceedings of LREC-06, 2006.

Ferrari S., Charnois, T., Jackiewicz A., Gardin P., Widlocher A., «Jugements d'évaluation : des constituants détachés à d'autres formes régulières ", $\sigma^{e}$ Journées $J L C$, Lorient, 10-12 septembre 2009. 
Franckel J.J., Lebaud D., «Diversité des valeurs et invariance du fonctionnement de en, préposition et préverbe ", Langue française $\mathrm{n}^{\circ}$ 91, 1991, p. 56-79.

Guimier C., Les adverbes du français, Ophrys, Collection L'essentiel Français, 1996.

Jackiewicz A., «L'évaluation à la périphérie du prédicat : constructions, lexiques et relations sémantiques ", Arena Romanistica $n^{\circ} 4$, Actes du $28^{e}$ Colloque international sur le lexique et la grammaire, 2009, Bergen, Norvège.

Jackiewicz A., Charnois T., Ferrari S., «Jugements d'évaluation et constituants périphériques ", TALN'09, Senlis, 2009a.

Jackiewicz A, Vigier D., Charnois T., Ferrari S., «Vers une analyse automatique des discours évaluatifs. Le cas des constituants détachés 'en $\mathrm{N}<\exp >^{\prime}$ "), LPTS 2009. Paris, 21-23 septembre 2009b.

Juliard J., La reine du monde, Flammarion, Café Voltaire, 2007.

Kerbrat-Orecchioni C., L'énonciation, Armand Colin, 2002.

Mann W.C., Thompson S.A., "Rhetorical Structure Theory: toward a functional theory of text organization", Text, vol. 8, n 3, 1988, p. 243-281.

Martin J.R., White P.R.P., The Language of Evaluation. Appraisal in English. Palgrave, 2005.

Neveu F., Etudes sur l'apposition. Aspects du détachement nominal et adjectival en français contemporain, dans un corpus de textes de J.-P. Sartre, Honoré Champion Editeur, Paris, 1998.

Neveu F. (éd) « Nouvelles recherches sur l'apposition », Langue française, n ${ }^{\circ} 125$, Larousse, 2000.

Noailly M., L'adjectif en français, Ophrys, Collection L'essentiel Français, 1999.

Pang B., Lee L., "A sentimental education: Sentiment analysis using subjectivity summarization based on minimum cuts", Proceedings of the $42^{\text {nd }}$ Annual Meeting of the $A C L, 2004$.

Pang B., Lee L., "Opinion Mining and Sentiment Analysis", Fondations and Trends in Information Retrieval, vol. 2, p.1-135, 2008.

Turney P.D., "Thumbs up or thumbs down? semantic orientation applied to unsupervised classification of reviews", Proceedings of the 40 ${ }^{\text {th }}$ Annual Meeting of the ACL, 2002.

Vigier D., «Contribution à une étude des constructions antéposées du type: 'En homme intelligent et humain, il partagea tout de suite l'inquiétude de Marcel' (J.Verne) », Discours, $\mathrm{n}^{\circ} 2,2008$.

Whitelaw C., Garg N., "Argamon S. Using appraisal taxonomies for sentiment analysis", Proceedings of MCLC-05, the $2^{\text {nd }}$ Midwest Computational Linguistic Colloquium. 2005.

Zerguini N., Etude des groupes adjectivaux extra-prédicatifs à valeur axiologique, Mémoire de Master 1 Informatique et Ingénierie de la Langue pour la Gestion de l'Information, Université de Paris-Sorbonne, 2009. 